\title{
Internal Friction of Metallic Glasses: Mechanisms and Conditions of their Realization
}

\author{
V.A. Khonik \\ Department of General Physics, State Pedagogical University, Lenin Str. 86, 394043 Voronezh, Russia
}

\begin{abstract}
The role of structural relaxation in the internal friction behaviour of metallic glasses is analysed. It is argued that this behaviour is uniquely determined by the structural relaxation rate during testing. Assumed damping mechanisms under "intensive" and "kinetically inhibited" structural relaxation are discussed.
\end{abstract}

\section{INTRODUCTION}

Internal friction (IF) is a sensitive and powerful method for investigation of structural rearrangements in solids. This method was started to be applied to metallic glasses (MG) in the beginning of the $70-\mathrm{s}$, soon after fabrication of the first $M G$. IF investigations of metallic glasses are being continued hitherto and a large experimental material is accumulated. The main experimental facts which require first and foremost interpretation consist of the following: i) a considerable and rapidly increasing with temperature damping at temperatures higher than the room one [1-5]; ii) low temperature $(\mathrm{T}<300 \mathrm{~K}$ ) relaxation IF peaks in MG underwent no preliminary treatment [6-10]; iii) low temperature relaxation IF peaks and hysteresis damping in plastically predeformed or hydrogenated MG [11-26]. In all cases IF is dependent on preliminary heat treatment. Therefore, one must conclude that spontaneous atomic rearrangements which take place in a glass at any temperature and are generally known as the "structural relaxation", influence the energy dissipation mechanisms. The purpose of the present paper consists of an analysis of assumed energy losses mechanisms taking into account a particular role of structural relaxation. We shall try to show that these mechanisms are determined by the structural relaxation rate during testing.

\section{SOME REMARKS ON THE KINETICS OF STRUCTURAL RELAXATION IN METALLIC GLASSES}

First of all it should be mentioned that structural relaxation in absence of external load results in no inelastic deformation, if one does not take into account nearly isotropic contraction of structure. An external stress leads to a distorsion of potential energy profiles of atomic shear rearrangements in particular regions of structure ("relaxation centres") and isolates the direction of predominant shearing. There arises a "directed structural relaxation", that is a structural relaxation oriented by external stress. This leads to accumulation of inelastic deformation according to orientation and magnitude of applied stress [27-29]. If structural relaxation proceeds at a high rate then sign-alternating loading results in viscoplastic IF and at frequencies low enough such an IF is dominating. But if structural relaxation is kinetically inhibited and during the loading half-period the 
mechanism of directed structural relaxation does not lead to a sufficient inelastic deformation then other IF mechanisms, outlined below, may come into action.

A natural question arises about the boundary between testing under "intensive" and "kinetically inhibited" structural relaxation. Meanwhile, this boundary is well determined because MG are being produced by quenching to room temperature and are stored at this temperature for a long time before testing. It is known that thermocycling below room temperature does not cause any sufficient irreversible property changes, while any heat treatment at a higher temperature gives rise to such changes. Therefore, one must conclude that structural relaxation below room temperature proceeds at an extremely low rate and it is this temperature that defines the boundary mentioned above.

A direct corroboration of this conclusion results from non-isothermal stress relaxation measurements which are generally known to be very sensitive to the rate of internal atomic rearrangements. At room and lower temperatures stress relaxation rate of loaded $\mathrm{MG}$ is extremely low but at $T>350-370 \mathrm{~K}$ its rapid increase is observed [30,31].

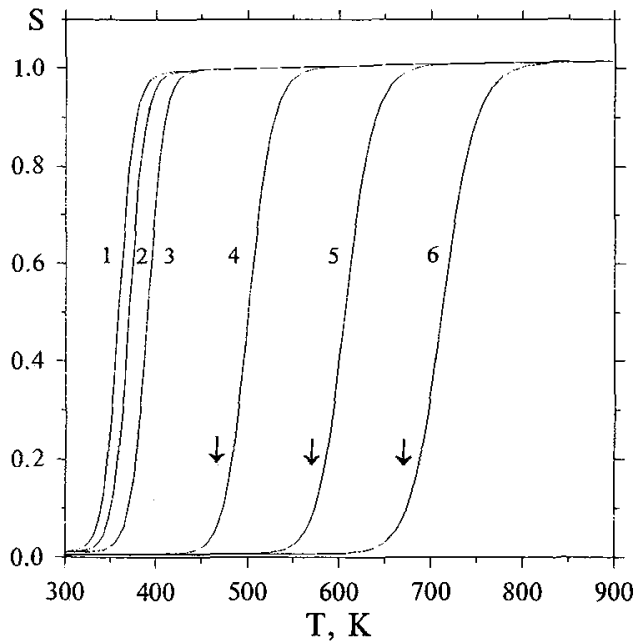

Figure 1: Calculated temperature dependences of the relative structural relaxation rate of a model sample after various heat treatments [33]: 1 - 3 - after preannealing at $\mathbf{T}_{\mathrm{a}}=293 \mathrm{~K}$ during one week (1), one month (2) and ten months (3); 4 - 6 after preannealing during $\tau=30 \mathrm{~min}$ at $\mathrm{T}_{\mathrm{a}}=473,573$ and $673 \mathrm{~K}$, correspondingly. Heating rate $-0.17 \mathrm{~K} / \mathrm{s}$. The arrows indicate preannealing temperatures $T_{a}$ for three last heat treatments. rapidly under cooling) and "intensive" ( $T>T_{a}$, structural relaxation rate is equal to that of the as-cast sample) structural relaxation. The $S(T)$ dependence shown in this Fig. is in excellent agreement with the non-isothermal stress relaxation measurements of samples preannealed at various $T_{a}[30,31,33,35]$.

MG production using standard melt spinning technique results naturally in the case $T_{a} \approx$ $300 \mathrm{~K}$. It is this temperature that defines for as-cast samples the approximate position of the boundary outlined above. It is important to note that the preannealing time at room temperature does not exert any sufficient influence on the position of this boundary. Such a conclusion can be drawn from curves 1 to 3 in Fig. 1 which correspond to room temperature preannealing times equal to one week, one month and ten months. 


\section{INTERNAL FRICTION UNDER INTENSIVE STRUCTURAL RELAXATION}

The main peculiarities of internal friction behaviour in this case can be illustrated by Fig.2 where IF temperature dependences of $\mathrm{MG} \mathrm{Co} \mathrm{C}_{70} \mathrm{Fe}_{5} \mathrm{Si}_{15} \mathrm{~B}_{10}$ measured at various frequencies and heating rates are shown. Curve 1 gives $\mathrm{Q}^{-1}(\mathrm{~T})$ dependence of as-cast sample measured with the help of the vibrating reed technique at $\mathrm{f} \approx 380 \mathrm{~Hz}$. The damping level increases

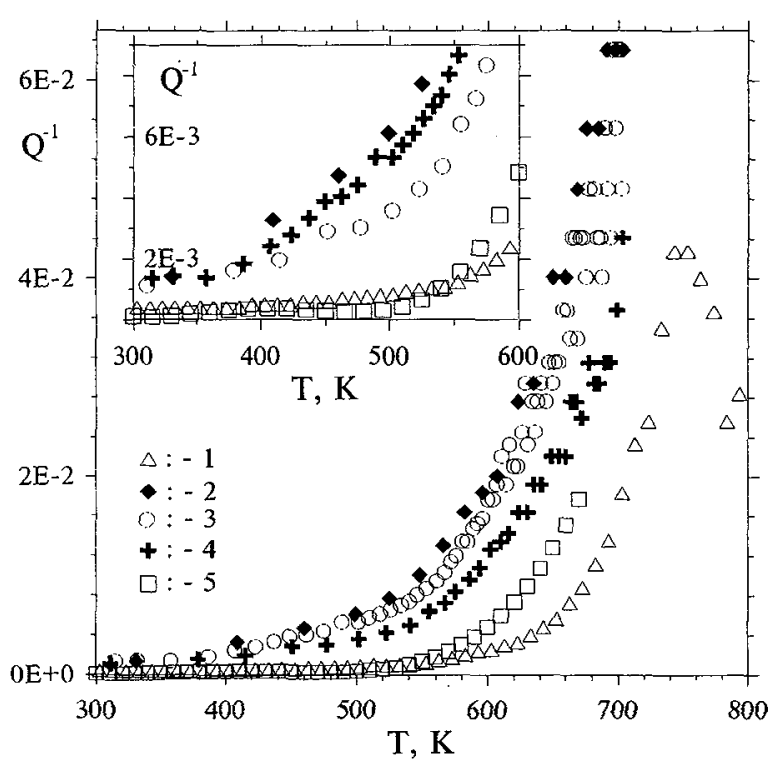

Figure 2: IF temperature dependences of $\mathrm{Co}_{70} \mathrm{Fe}_{5} \mathrm{Si}_{15} \mathrm{~B}_{10}$ MG measured using vibrating reed technique $(1, \mathrm{f} \approx 380$ $\mathrm{Hz}$, heating rate $\dot{\mathrm{T}}=0.08 \mathrm{~K} / \mathrm{s})$ and torsion micropendulum [35] $(2-5, f=0.5 \div 1.5 \mathrm{~Hz}, \dot{T}=0.15$, 0.093 and $0.040 \mathrm{~K} / \mathrm{s}$ for the curves $2-4$, correspondingly). Curve 5 is measured under cooling after preannealing at $\mathrm{T}$ $=703 \mathrm{~K}$ during $30 \mathrm{~min}$. The insert shows the initial portion of $Q^{-1}(T)$ in an enlarged scale. Magnetomechanical damping is negligible. rapidly with temperature, reaching a maximum near the crystallization temperature $T_{c}$. It is generally accepted that this "crystallization" peak should be interpreted as IF background increasing with temperature and reflecting thermoactivated rearrangements in the amorphous state, and a cut-off of this background due to crystallizationinduced disappearance of relaxation sources [36,37].

Curves 2 to 4 give $Q^{-1}(T)$ at various heating rates obtained for the same MG using torsion micropendulum (f $0.5 \div 1.0 \mathrm{~Hz}$ ). It is seen, firstly, that a decrease of frequency leads to a large damping increase and, secondly, that the IF level increases with heating rate. Curve 5 gives IF temperature dependence obtained at the same frequency under cooling after preannealing at $T_{a}=703 \mathrm{~K}$ during 30 min. The choice of this heat treatment was determined, on the one hand, by the attempt to attain the highest possible relaxed state and, on the other hand, to avoid crystallization. A comparison of curves 2 to 5 shows that preannealing within the amorphous state decreases the IF level significantly. Taking this fact into account, two constituents in the IF of as-cast specimens can separated out: losses $Q_{S R}^{-1}$ due to structural relaxation during testing and losses $Q_{R E L}^{-1}$ caused by thermoactivated rearrangements in the relaxed amorphous structure. The first component can be determined by taking the difference between IF measured during the first heating run and that after preannealing: $Q_{S R}^{-1}(T, t)=Q^{-1}(T, t)-Q_{R E L}^{-1}(T)$ (see also Fig.4).

Two mechanisms can be proposed for the first constituent, $\mathrm{Q}_{\mathrm{SR}}^{-1}$ : A) reversible atomic rearrangements (reversible transitions in two-level relaxation centres with low or zero asymmetry $\Delta$ of the potential, Fig.3,a) which under sign-alternating stress result in anelastic behaviour (the corresponding rheological model is shown in the upper part of Fig.3,a) and define IF proportional to the concentration of relaxation centres. Correspondingly, the $Q_{S R}^{-1}$ level is determined by the degree of structural relaxation. The attempts $[38,39]$ undertaken to develop analytical models of such kind, however, did not allow to obtain equations correspondent to the experimental regularities. B) irreversible atomic rearrangements which occur only once in relaxation centres with large asymmetry of the potential, Fig.3,b. A set of 

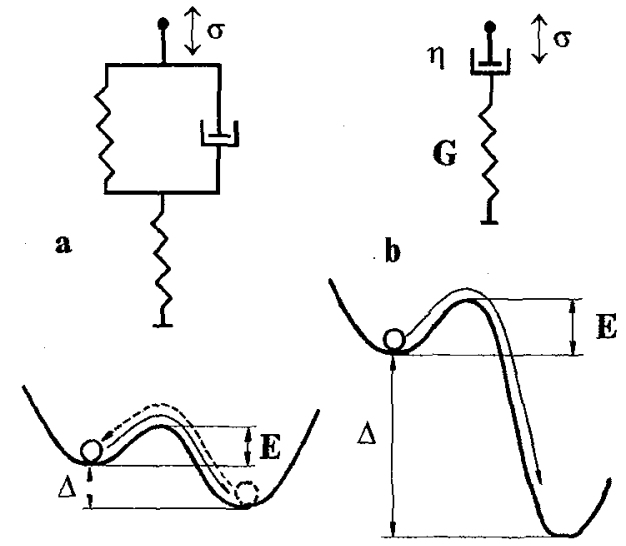

Figure 3: A schematic illustration of possible losses mechanisms in $M G$ under intensive structural relaxation. a) Two level relaxation centres with low or zero asymmetry $\Delta$ which define anelastic losses according to the model of standard anelastic body. b) Two level relaxation centres with latge asymmetry. Atomic rearrangements in such centres occur only once resulting in viscoplastic Maxwelltype losses. $\mathrm{E}$ is the activation energy of elementary atomic rearrangements.

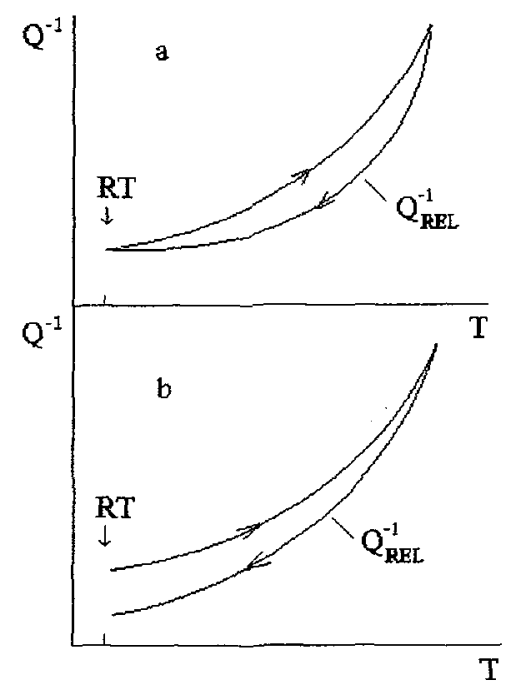

Figure 4: A schematic drawing of two possible cases of IF behaviour of MG at temperatures higher than the room one. A closed IF loop (a) corresponds to the relaxation mechanism $B$ and an opened IF loop (b) is realized when both mechanisms, A and B, or only the first of them, are operating. Room temperature (RT) is indicated.

such rearrangements in sign-alternating stress field results in viscoplastic IF according to the rheological model shown in the upper part of Fig.3,b. Such an IF is proportional to the rate of decrease of relaxation centres concentration, i.e.- proportional to the rate of structural relaxation.

Obviously, both mechanisms are operating in a real glass and their contributions are expected to be frequency dependent. One can propose a simple criterion which provides an information what mechanism is dominating. If $\mathrm{Q}_{\mathrm{SR}}^{-1}$ is determined only by the mechanism $\mathrm{B}$ then the IF loop "room temperature $\rightarrow$ heating to $T<T_{c} \rightarrow$ cooling to room temperature" is closed $\left(Q^{-1}\right.$ values at room temperature before and after annealing coincide, Fig. $4, a)$ because the structural relaxation rate at room temperature is extremely low both for as-cast and (in particular) preannealed samples. Consequently, this mechanism does not contribute in damping at room temperature. An opened IF loop (Fig.4,b) indicates the presence of both mechanisms, A and B, or only of the first of them. The difference between IF levels before and after heating in this case reflects the change of relaxation centres concentration as a result of heat treatment.

In a real experiment both closed and opened IF loops are observed. For example, Morito [40] obtained completely closed loops for $\mathrm{Fe}_{32} \mathrm{Ni}_{36} \mathrm{Cr}_{14} \mathrm{P}_{12} \mathrm{~B}_{6}$ at $\mathrm{f} \approx 0.4 \mathrm{~Hz}$. Fig. 2 shows the second case: IF levels at room temperature before and after heating do not coincide. However, the difference between these levels is quite small indicating a small contribution of the mechanism $A$ in $Q_{S R}^{-1}$.

It is generally known that structural relaxation of as-cast $M G$ is predominantly irreversible and reversible relaxations phenomena are observed, as a rule, after preliminary annnealing. Consequently, one should expect that IF of as-cast samples is mainly viscoplastic, at least, if frequencies are low enough. Anelastic losses should be observed mainly in annealed state or at high frequencies. 
The first quantitative model of viscoplastic IF due to structural relaxation (the mechanism B) was developed in $[29,41]$ and an expression for $Q_{S R}^{-1}$ was derived:

$$
\mathrm{Q}_{\mathrm{SR}}^{-1}=\mathrm{G} / \eta \omega
$$

where $G$ is the unrelaxed shear modulus, $\omega$ the circular frequency, $\eta$ the time/temperature dependent viscosity. Under linear heating,

$$
\eta^{-1}(\mathrm{~T})=\mathrm{N}_{0}\left(\mathrm{E}_{0}(\mathrm{~T})\right)_{2 \mathrm{CAT}}
$$

where $\dot{T}$ is the heating rate, $A=$ const $\approx 3 \cdot 1 \cdot 10^{-3} \mathrm{eV} / \mathrm{K}, \mathrm{N}_{0}$ the concentration of relaxation centres per unit activation energy interval, $\Omega$ the volume enclosed by an elementary structural relaxation event, $C$ the parameter which takes into account orienting influence of external stress on these events. The quantity $\mathrm{N}_{0}$ is temperature dependent via temperature dependence of the characteristic energy $\mathrm{E}_{0}$, which is determined as the energy correspondent to the maximal rate of relaxation centres decrease. It is seen from (1) and (2) that at the given temperature $\mathrm{Q}_{\mathrm{SR}}^{-1}$ is increasing with heating rate and decreasing with frequency, that is really observed in the experiment (see Fig.2 and [3,42-44]). Moreover, isothermal cuts of $\mathrm{Q}_{\mathrm{SR}}^{-1}(\mathrm{~T})$ curves at various heating rates must be straight lines in the $\mathrm{Q}_{\mathrm{SR}}^{-1} \cdot \omega-\dot{\mathrm{T}}$ co-ordinates with temperature dependent angle coefficients, in accordance with the experiment [41].

At the constant temperature the viscosity time dependence is given by

$$
\eta^{-1}(t)=k \operatorname{kN}_{0}\left(\mathrm{E}_{0}(\mathrm{t})\right) \Omega \mathrm{C} /(\mathrm{t}+\tau),
$$

where $k$ is the Boltzman's constant, $\tau=k T /$ TA the effective preannealing time. The $N_{0}(t)$ dependence involved in the Eq.(3) can be determined from IF measurements on linear heating using Eq.(1) [29,41]. Then it follows from Eqs (1) and (3) that isothermal IF dependences must be straight lines in the $\mathrm{Q}_{S \mathrm{R}}^{-1}-\mathrm{N}_{0}(\mathrm{t}) \Omega \mathrm{C} /(\mathrm{t}+\tau)$ co-ordinates with angle coefficients equal to $\mathrm{kT} / \omega$. Such a situation is really observed under low frequency measurements $[29,41]$.

An estimation [29] has shown that this viscoplastic mechanism is dominating in as-cast MG at frequencies $\omega \leq 10 \mathrm{~s}^{-1}$. Obviously, at higher $\omega$ the anelastic component caused by reversible transitions in two-level relaxation centres will increase. Therefore, an analysis of damping mechanisms at various frequencies promises new valuable information about reversible and irreversible relaxations and their relationship in as-cast and annealed states. At present these questions, as well as the nature of the $\mathrm{Q}_{\mathrm{REL}}^{-1}$ constituent, are poorly investigated.

\section{INTERNAL FRICTION UNDER KINETICALLY INHIBITED STRUCTURAL RELAXATION}

\subsection{Internal Friction in As-Cast Samples}

It was mentioned above that low and rapidly decreasing under cooling structural relaxation rate is realized at $\mathrm{T} \leq 300 \mathrm{~K}$. Hence, it can be stated that viscoplastic damping mechanism is ineffective in this temperature range. Generally, one can suppose that the mechanism of anelastic losses can contribute significantly to the measured damping. The experiment shows, however, that it is not the case.

This conclusion can be drawn from Fig.5 where low temperature IF and normalized elastic modulus dependences for eight MG of different compositions (metal-metal, metalmetalloid, with hydride-forming elements and without them) are shown. In all cases $\mathrm{Q}^{-1}$ is quite low and weakly increases with temperature whereas normalized modulus slightly decreases with temperature rise indicating an absence of thermoactivated atomic rearrangements. This behaviour was pointed out in a number of papers $[20,48,49]$. Approximately a linear IF increase with temperature allows to suppose that thermoelastic damping is the dominating mechanism of losses [47] and a monotonic modulus decrease should be attributed to the usual anharmonism. 


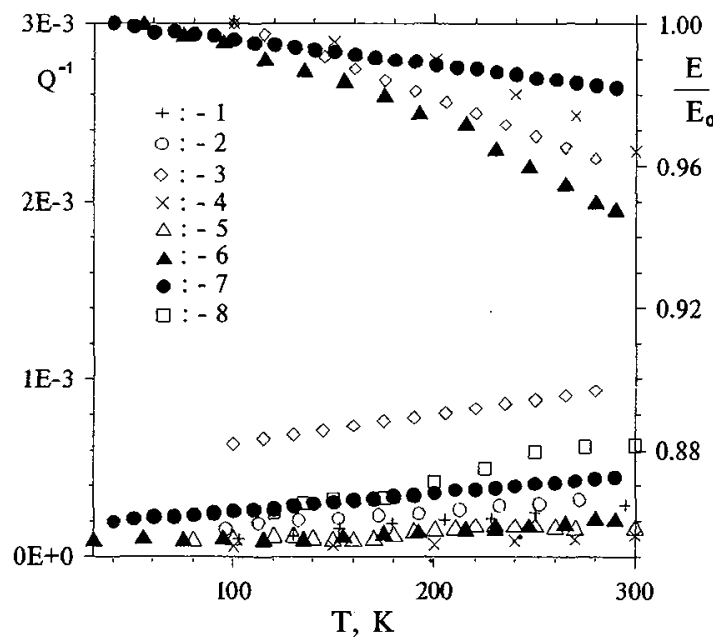

Figure 5: Low temperature IF and normalized elastic modulus dependences of as-cast MG measured by the vibrating reed technique. $1-\mathrm{Fe}_{32} \mathrm{Ni}_{30} \mathrm{Cr}_{14} \mathrm{P}_{12} \mathrm{~B}_{6}(64$ $\mathrm{Hz}$ ) [18]; $2-\mathrm{Pd}_{80} \mathrm{Si}_{20}[18] ; 3-\mathrm{Ni}_{60} \mathrm{Zr}_{40}(45 \mathrm{~Hz})$ [45]; $4-\mathrm{Co}_{70.4} \mathrm{Fe}_{4.6} \mathrm{Si}_{15} \mathrm{~B}_{10}[46] ; 5-\mathrm{Pd}_{77.5} \mathrm{Cu}_{6} \mathrm{Si}_{16.5}(200$ $\mathrm{Hz})[11] ; 6-\mathrm{Cu}_{50} \mathrm{Ti}_{50}(200 \mathrm{~Hz})$ [12]; $7-\mathrm{Ni}_{60} \mathrm{Nb}_{40}$ $(300 \mathrm{~Hz})$ [17]; $8-\mathrm{Ni}_{78} \mathrm{Si}_{8} \mathrm{~B}_{14}(280 \mathrm{~Hz})$ [47].

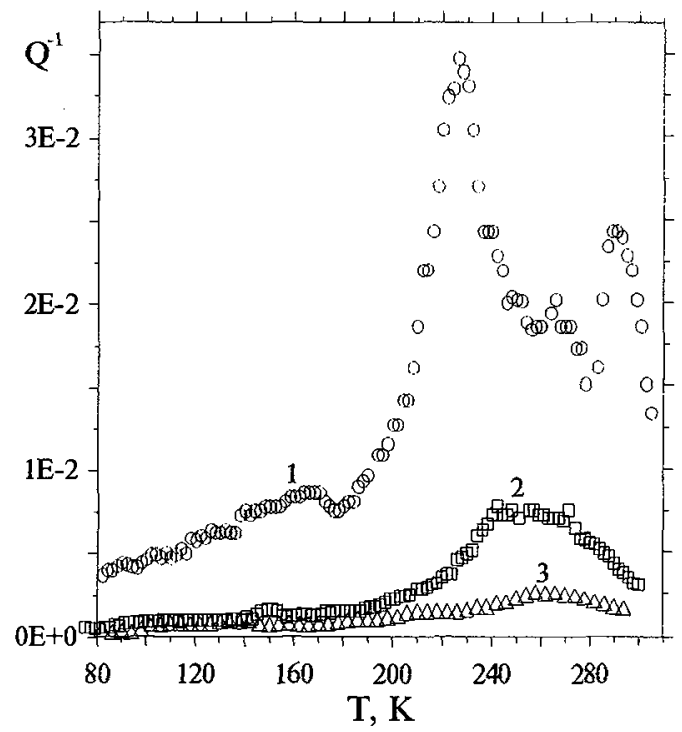

Figure 6: Low temperature IF of rolled $\mathrm{Ni}_{78} \mathrm{Si}_{8} \mathrm{~B}_{14}$ and its evolution with rolling degree. 1 - 3 - rolled by $2,3.6$ and $4.9 \%$, respectively. Strain amplitude $\varepsilon_{0}=7 \cdot 10^{-6}, \mathrm{f} \approx 290 \mathrm{~Hz}$.

However, in Refs [6-10,47] were described large relaxation peaks in a number of MG not subjected to any preliminary treatment. It should be pointed out in this connection that the authors $[4,16,50,51]$ especially underlined that damping level can be critically dependent on the conditions of samples gripping. A special investigation has shown [17] that inappropriate gripping results in plastic deformation of samples and, due to this fact, in appearance of large IF peaks analogous to those described below, in Sec.4.2. However, sometimes without special experimental efforts it is impossible to determine whether these peaks are characteristic of as-cast or plastically deformed samples. Therefore, the above mentioned data about low temperature IF peaks in initial samples should be considered as not verified in the meanwhile.

Hence, there is good reason to believe that under the conditions of kinetically inhibited structural relaxation any effective mechanisms of energy dissipation in carefully gripped samples are absent, at least, in the range $20<\mathrm{T}<300 \mathrm{~K}$ and at frequencies $\mathrm{f}<10^{3} \mathrm{~Hz}$.

\subsection{Internal Friction in Plastically Deformed Samples}

The transition from intensive to kinetically inhibited structural relaxation results in cardinal change of plastic deformation mechanism, from homogeneous viscoplastic flow induced by directed structural relaxation to inhomogeneous elastic-plastic one [52] which leads to formation of slip bands quite analogous to those in crystals. The authors [11] were the first who showed that inhomogeneous deformation results in qualitative change of low temperature IF. Later this conclusion was confirmed and supplemented with a number of details [12-17]. In summarised view the obtained results can be briefly formulated as follows. Inhomogeneous deformation leads to appearance of large relaxation IF peaks in the range $200<\mathrm{T}<300 \mathrm{~K}$ with the activation energy of about $0.5 \mathrm{eV}$ and the preexponential factor close to the Debye frequency. An example of such a peak for $\mathrm{Ni}_{78} \mathrm{Si}_{8} \mathrm{~B}_{14}$ is shown in Fig.6. Deformation-induced IF peaks can be suppressed by high prestrain (curves 1 to 3 , Fig.6), heat treatment within the amorphous state or irradiation (see below, curves 2 and 3 in Fig.8). It was shown that damping at the temperatures below the peak temperature (i.e. at $\mathrm{T}<150 \div 200 \mathrm{~K})$ is hysteresis and at the higher temperatures hysteresis background is 


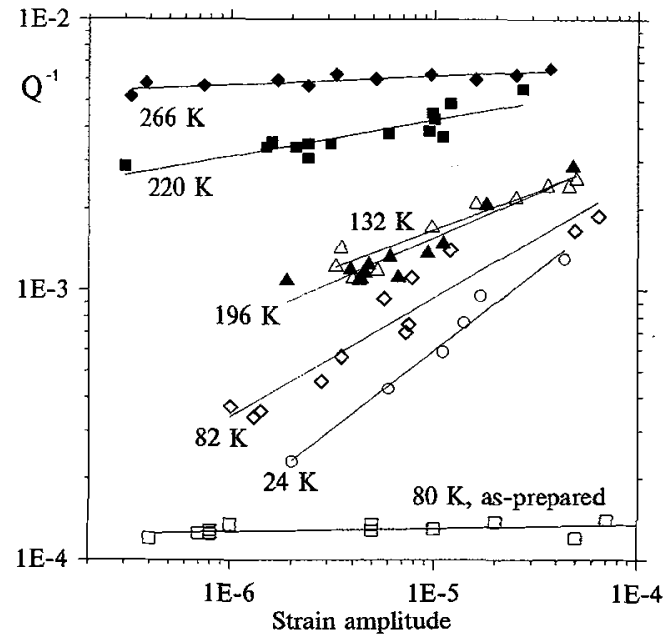

Figure 7: IF amplitude dependences of $\mathrm{Ni}_{78} \mathrm{Si}_{8} \mathrm{~B}_{14} \mathrm{MG}$ in the initial and predeformed states at various temperatures. Measurements are carried out using the specimen with the IF spectrum shown by the curve 2 in Fig.6.

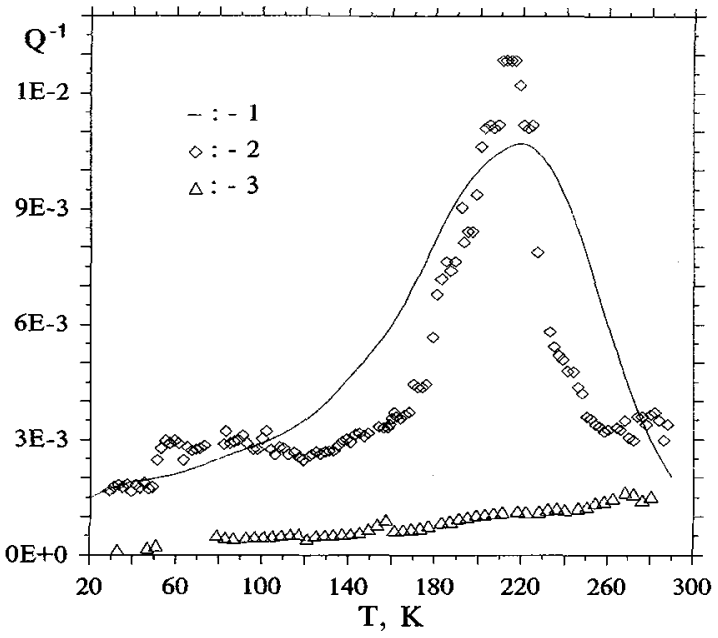

Figure 8: Low temperature IF of $\mathrm{Cu}_{50} \mathrm{Ti}_{50}$ MG. 1 after electrolytic hydrogenation by $\mathrm{C}_{\mathrm{H}}=3.3$ at.\% ( $\mathrm{f} \approx$ $\left.500 \mathrm{~Hz}, \varepsilon_{0} \propto 10^{-6}\right)$ [59]; after rolling by $2.8 \%(2$, $\varepsilon_{0}=6 \cdot 10^{-5}, \mathrm{f} \approx 300 \mathrm{~Hz}$ ) and subsequent $2 \mathrm{MeV}$ electron irradiation of the same specimen by the fluence $1 \cdot 10^{19} \mathrm{~cm}^{-2}\left(3, \varepsilon_{0}=5 \cdot 10^{-6}, \mathrm{f} \approx 300 \mathrm{~Hz}\right)[14]$.

superimposed by a relaxation IF peak. It was discussed in detail earlier [12-17] that these characteristic peculiarities closely resemble behaviour of crystals with fresh mobile dislocations and, most probably, reflect dislocation-like character of $\mathrm{MG}$ inhomogeneous flow (see also [53-55]). Therewith the deformation-induced IF peak is, obviously, a direct analogue of the Hasiguti peaks in crystals.

A salient feature of IF in predeformed MG can be illustrated by Fig.7. In the initial state the amplitude dependent IF is absent (see also [11]) indicating a lack of (mobile) dislocation-like defects. Inhomogeneous deformation results in appearance of IF amplitude dependence that should be interpreted as occurrence of linear dislocation-like defects, not characteristic of the as-cast state. Fig.7 shows that in the double logarithmic co-ordinates IF amplitude dependences turn out to be straight lines pointing up that the functional IF expression has the form $Q^{-1}\left(\varepsilon_{0}, T\right)=\varphi(T) \cdot \varepsilon_{0}^{n}$, where $\varphi(T)$ is some temperature dependent function, $\mathrm{n}$ the dimensionless constant of about $0.5 \div 0.6$ in the range $\mathrm{T}<160 \div 180 \mathrm{~K}$, where hysteresis damping is predominant. The analogous situation is realized in the case of dislocation hysteresis in crystals [56]. One more interesting detail should be also emphasized: as different from crystals, any amplitude independent IF in predeformed samples is not detected even at very low strain amplitudes, $\varepsilon_{0} \sim 10^{-7}$. Probably, this fact indicates presence of long dislocation-like segments in deformed structure.

Inhomogeneous flow can arise not only under rolling or improper gripping but also during hydrogenation. It is known (see Refs. in [17]) that hydrogen can accumulate in voids and cracks under pressure (up to $10^{3} \mathrm{MPa}$ ) that is high enough to activate local microplastic deformation. Microplastic flow can arise also via hydride growth (due to the difference of specific volumes of matrix and growing hydrides) on the background of weakening of interionic metal-metal bonding due to hydrogen adsorption [17]. The effect of hydrogenation-induced $\mathrm{MG}$ plasticity $[57,58]$ clearly indicates such possibilities. In the present author's opinion, the widely spread statements, that IF peaks obtained after hydrogenation are due to single hydrogen atom jumps and are a kind of Snoek relaxation, are barren of experimental basis. Hydrogen seems to be only an indirect reason of IF peak appearance and the direct reason consists of inhomogeneous microplastic deformation of structure during hydrogenation. 
This conclusion can be drawn, for example, from a comparison of curves 1 and 2 in Fig.8, one of which corresponds to IF temperature dependence of a rolled, the other - of a hydrogenated $\mathrm{Cu}_{50} \mathrm{Ti}_{50}$ sample. Clear similarity of these dependences suggests a similarity of underlying relaxation mechanisms. This statement was argued in Ref. [17] in detail where IF temperature dependences of rolled and hydrogenated $\mathrm{Ni}_{60} \mathrm{Nb}_{40}$ samples were measured at identical experimental conditions. It was shown that both rolling and hydrogenation result in relaxation IF peaks on a hysteresis background, the shapes of the peaks and their temperature positions closely resemble and the activation energies are identical. In both cases IF peaks are corresponded to a large activation volume, inconsistent with an assumption about point-like character of defects responsible for relaxation. As a whole, the results [17] confirms the proposal about unity of nature of dissipation mechanisms in cold deformed and hydrogenated MG. Further investigations in this field, however, seem to be desirable.

The above-mentioned clearly indicates that dislocation-like defects can exist in predeformed MG. Therefore, some comments on the nature of these defects can be pointed out.

\subsection{On the Origin of Dislocation-like Defects in Metallic Glasses}

The nature of dislocation-like defects in glassy structures is a subject of discussions. One of the main questions is that about the character of stability of these defects. Two cases can be considered: i) dislocations present in the as-cast state of glass and are topologically stable (like dislocations in crystals), ii) dislocations appear only as a result of loading and are stable only in the kinetic sense. The first case means that dislocations can disappear only on specimen surfaces or due to annihilation but cannot be removed by any set of local atomic displacements. Such a situation can be easily envisioned within the framework of the disclination models of non-crystalline structures (see [60] and Refs. therein). Within this approach MG is modelled as a medium with high density of topologically stable defects disclinations. A disclination dipole is equivalent to a dislocation $[60,61]$ and dislocations are, therefore, topologically stable as well. Disclination dipoles - dislocations - are considered to be carriers of plastic deformation of MG [60,62,63]. Two ways of dislocation density increase under loading are possible: generation of dislocations due to stress-induced displacements of disclinations and nucleation of dislocations on free surfaces near stress concentrators. Under the condition of kinetically inhibited structural relaxation (i.e. when disclination network is not affected by thermal fluctuations) these dislocations can determine, in principle, hysteresis damping and low temperature IF anomalies described above. However, the degree of adequacy of this approach is not clear. In particular, it is not evident whether a structure with high dislocation density (and, correspondingly, with short mobile dislocation segments) which appears as a result of predeforming can give rise to hysteresis IF observed in the experiment.

If, actually, the as-cast state is free of dislocation-like defects, then these latter can appear as a result of loading. Such a situation was modelled in Ref. [64] where dislocations were introduced into MG computer model using standard continual procedures. It was shown that after moderate heat treatment dislocations can be detected in the model as linear singularities of the internal stress field. However, high temperature annealing results in complete disappearance of these defects as localized singularities due to spread of the dislocation displacement field throughout the whole specimen. This experiment clearly indicates that dislocation-like defects can exist in real MG as kinetically stable defects which can determine low temperature IF anomalies discussed above only if the loading half period is much smaller than the time constant of structural relaxation. This situation seems to be most likely.

In either case, further theoretical and experimental investigations are needed in order to understand the nature of "enigmatic entities" [65] - dislocations in glasses. 


\section{CONCLUSIONS}

1. Structural relaxation rate exerts primary control over type and level of damping in metallic glasses. One must distinguish between internal friction testing under "intensive" and "kinetically inhibited" structural relaxation. The boundary between these types of testing is quite distinct and is determined by preliminary heat treatment of a glass.

2. Testing under "intensive" structural relaxation is realized for as-cast metallic glasses at T

$>300 \mathrm{~K}$. In this case it is possible: i) Anelastic internal friction determined by reversible atomic rearrangements in relaxation centres which constitute two level systems with small asymmetry of the potential. Damping of such a type is proportional to the concentration of relaxation centres and, consequently, to the degree of structural relaxation of glass. ii) Viscoplastic internal friction occurring as a result of irreversible rearrangements in relaxation centres with large asymmetry of the potential. Damping of this type is proportional to the rate of decrease of relaxation centres concentration, i.e. - to the rate of structural relaxation. At low frequencies viscoplastic damping is predominant.

3. Testing under "kinetically inhibited" structural relaxation is realized for as-cast samples at $\mathrm{T}<300 \mathrm{~K}$. Internal friction temperature dependences have no peculiarities in this case.

4. Plastic flow of metallic glasses under "kinetically inhibited" structural relaxation proceeds, obviously, via a dislocation-like mechanism. Samples predeformed in such a way (by forging, rolling of hydrogenation) display specific hysteresis and relaxation damping, analogous to that in dislocated crystals.

\section{Acknowledgements}

The author is grateful to the ICIFUAS 11 Organizing Committee for the financial support for participating in the Conference. This work was partially supported by the Russian Foundation of Basic Research under grant N 95-02-03970. Dr. V.V.Makoukha and the PGPT Foundation are also greatly acknowledged.

\section{References}

[1] Chen H.S., Leamy H.J., Barmatz M., J.Non-Cryst.Solids 5 (1971) 444-448.

[2] Soshiroda T., Koiwa M., Masumoto T., J.Non-Cryst.Solids 22 (1976) 173-187.

[3] Tuagi S., Lord A.E., J.Non-Cryst.Solids 30 (1979) 273-283.

[4] Morito N., Mater.Sci.Eng. 60 (1983) 261-268.

[5] Sinning H.-R., Haessner F. Scr.Metall. 20 (1986) 1541-1546.

[6] Barmatz M., Chen H.S. Phys.Rev.B. 9 (1974) 4073-4083.

[7] Yoon H.N., Eisenberg A. J.Non-Cryst.Solids 29 (1978) 357-364.

[8] Berry B.S., Pritchet W.C., Tsuei C.C. Phys.Rev.Lett. 41 (1978) 410-413.

[9] Kunzi H.U., Ageyman K., Gunterodt H.-J. Solid State Commun. 32 (1979) 711-714.

[10] Kalinin Yu.E., Zolotukhin I.V. Fiz.Tverd.Tela 22 (1980) 223-224.

[11] Zolotukhin I.V., Belyavsky V.I., Khonik V.A. Fiz.Tverd.Tela 27 (1985) 1788-1794.

[12] Belyavsky V.I., Khonik V.A.Ryabtseva T.N. Metallofizika 11 (1989) 106-111.

[13] Zolotukhin I.V., Belyavsky V.I., Khonik V.A., Ryabtseva T.N. Fiz.Metall.Metalloved. 68 (1989) 185-191.

[14] Zolotukhin I.V., Belyavsky V.I., Khonik V.A., Ryabtseva T.N. Phys.Stat.Solids(a) 116 (1989) 255-265.

[15] Khonik V.A., Safonov I.A., Ryabtseva T.N. Fiz.Tverd.Tela 35 (1993) 2568-2575.

[16] Khonik V.A. J.Alloys Compounds 211/212 (1994) 114-117.

[17] Khonik V.A., Spivak L.V. Acta Mater. 44 (1996) 367-381.

[18] Berry B.S., Pritchet W.C. Scr.Metall. 15 (1981) 637-642.

[19] Berry B.S., Pritchet W.C. Phys. Rev.B 24 (1981) 2299-2302.

[20] Yoshinary O., Koiwa M., Inoue A., Masumoto T. Acta Metall. 31 (1983) 2063-2072.

[21] Hazelton L.E., Johnson W.L. J.Non-Cryst.Solids 61-62 (1984) 667-672.

[22] Stolz U., Weller M., Kirchheim R. Scr.Metall. 20 (1986) 1361-1366. 
[23] Mizubayashi H., Katoh Y., Okuda S. Phys.Stat.Solids (a) 104 (1987) 469-485.

[24] Berry B.S., Pritchet W.C. Z.Phys. Chem. 163 (1989) 381-390.

[25] Sinning H.-R., Nicalaus M., Haessner F. Sct.Metall. 23 (1989) 471-476.

[26] Sinning H.-R. J.Alloys Compounds 211/212 (1994) 216-221.

[27] Kosilov A.T., Khonik V.A., Izv.RAN. Ser.Fiz. 33 (1993) 3393-3399.

[28] Khonik V.A., Kosilov A.T., Mikhailov V.A., J.Non-Cryst.Solids 192-193 (1995) 420423.

[29] Belyavsky V.A., Bobrov O.P., Kosilov A.T., Khonik V.A., Fiz.Tverd.Tela 38 (1996) 30-40.

[30] Dzuba G.A., Zolotukhin I.V., Kosilov A.T., Khonik V.A. Fiz.Tverd.Tela 33 (1991) 33933399.

[31] Khonik V.A., Kosilov A.T. J.Non-Cryst.Solids 170 (1994) 270-277.

[32] Girt E., Knezevic G., Baltic R., Girt Er., Bikic E.. Mater.Sei.Eng. A133 (1991) 541-543.

[33] Bobrov O.P., Kosilov A.T., Khonik V.A. Fiz.Metall.Metalloved. 81 (1996) 123-132.

[34] Bobrov O.P., Kosilov A.T., Mikhailov V.A., Khonik V.A. Izv.RAN. Ser.Fiz., 1996, in press.

[35] Bobrov O.P., Kosilov A.T., Khonik V.A. Fiz.Tverd.Tela, 1996, in press.

[36] Hettwer K.J., Haessner F. Mater.Sci.Eng. 52 (1982) 147-154.

[37] Sinning H.-R. J.Non-Cryst.Solids 110 (1989) 195-202.

[38] Morito N., Egami T. Acta Metall. 32 (1984) 603-613.

[39] Chen H.S., Morito N. J.Non-Cryst.Solids 72 (1985) 287-299.

[40] Morito N. Mater.Sci.Eng. 60 (1983) 261-268.

[41] Belyavsky V.I., Bobrov O.P., Kosilov A.T., Khonik V.A. This Conference, p.

[42] Sinning H.-R., Haessner F. J.Non-Cryst.Solids 93 (1987) 53-66.

$143]$ Sinning H.-R., Haessner F. Z.Phys.Chem. 156 (1988) 115-121.

[44] Myung W.-N., Park K.-H., Jang D.-H. J.Non-Cryst.Solids 192-193 (1995) 401-404.

[45] Berry B.S., Pritchet W.C. Mater.Sci.Eng. 97 (1988) 419-425.

[46] Kunzi H.U., Ambruster E., Agyeman K. " Relaxation and internal friction in metallic glasses", Conf. Metall. Glass: Sci. and Technol. Budapest, 1980, Proc. Vol.1. (Budapest, 1980) pp.107-117.

[47] Sinning H.-R., Woldt E., Haessner F. Mater.Sci.Eng. 97 (1988) 501-504.

[48] He S., Liu M., Tang L. J.Sichuan Univ.Nat.Sci. 26 (1989) 437-441.

[49] Mizubayashi H., Matsumoto M., Okuda S. J.Alloys Compounds 211/212 (1994) 237-240.

[50] Hettwer K.J., Haessner F. Mater.Sci.Eng. 52 (1982) 147-154.

[51] Okumura K., Ho S.C., Inoue A., Masumoto T. J.Non-Cryst.Solids 130 (1991) 304-310.

[52] Bobrov O.P., Khonik V.A., Safonov I.A. Scr.Metall.Mater. 32 (1995) 1369-1373.

[53] Kosilov A.T., Khonik V.A. Ryabtseva T.N. Metallofizika 12 (1990) 37-41.

[54] Zolotukhin I.V., Kosilov A.T., Khonik V.A., Ryabtseva T.N., Lukin A.A., Prokoshina G.F. Fiz.Tverd.Tela 32 (1990) 1378-1384.

[55] Bobrov O.P., Khonik V.A. J.Non-Cryst. Solids 192-193 (1995) 605-607.

[56] Blanter M.S., Golovin I.S., Golovin S.A., Il'in A.A., Sarrak V.I. Mechanical spectroscopy of metallic materials (Int.Eng.Acad.Publ., Moscow, 1994) 254 pp.

[57] Spivak L.V., Khonik V.A., Skryabina N.E. Pisma v Zhum.Tekhn.Fiz. 19 (1993) 39-43.

[58] Spivak L.V., Khonik V.A., Skryabina N.E. Zhum.Tekhn.Fiz. 65 (1995) 104-113.

[59] Mizubayashi H., Agari H., Okuda S. Z.Phys. Chem. 163 (1989) 391-396.

[60] Ovid'ko I.A. Defects in condensed matter: glasses, crystals, quasicrystals, liquid crystals, magnetics, superfluid liquids (Znanie, St.Petersburg, 1991) pp.1-52.

[61] Vladimirov V.1., Romanov A.E. Disclinations in crystals (Nauka, Leningrad, 1986) pp.5164.

[62] Ovid'ko I.A. Pisma v Zhurn.Tekhn.Fiz. 13 (1987) 443-446.

[63] Ovid'ko I.A. Phil.Mag. B. 59 (1989) 523-534.

[64] Chaudhari P., Spaepen F., Steinhardt P.J. "Defects and atomic transport in metallic glasses", Glassy metals II, Topics in Applied Physics, Beck H. and Gunterodt H.-J. Eds. (Mir, Moskva, 1986) pp.151-198.

[65] Gilman J.J. J.Appl.Phys. 44 (1973) 675-679. 